\title{
Immunohistochemical and ultrastructural localization of prostaglandin $H$ synthase in the preimplantation mouse embryo
}

\author{
R. M. F. van der Weiden ${ }^{1}$, L. J. Wisse ${ }^{2}$, F. M. Helmerhorst ${ }^{1}$, \\ M. J. N. C. Keirse ${ }^{1}$ and R. E. Poelmann ${ }^{2}$ \\ ${ }^{1}$ Department of Obstetrics and Gynecology, and ${ }^{2}$ Department of Anatomy and Embryology, Leiden \\ University Hospital, Leiden, The Netherlands
}

\begin{abstract}
The immunohistochemical and ultrastructural localization of prostaglandin $\mathrm{H}$ synthase (PGH synthase) was studied in the Albino Swiss CF-1 mouse at different stages of embryonic development (two-cell stage, four-eight cell stage, morula and blastocyst). Flushed embryos and sections of uteri and oviducts containing embryos were treated with a mouse IgG monoclonal anti-PGH synthase antibody. The second antibody (rabbit anti-mouse) was conjugated with peroxidase or fluorescein isothiocyanate for light microscopy, fluorescence microscopy and confocal scanning. For reflection contrast microscopy and transmission electron microscopy a second antibody, goat anti-mouse, was conjugated with ultrasmall gold particles. Controls without anti-PGH synthase were used concurrently. All embryos demonstrated PGH synthase reactivity. Immunostaining appeared to be more intense at the two-cell stage, four-eight cell stage embryos and morulae than in blastocysts. Further examination indicated an intracytoplasmic location for PGH synthase, which was confirmed by stereoscopic photographs made during confocal scanning microscopy and by the immunostaining patterns observed with reflection contrast microscopy and transmission electron microscopy. Transmission electron microscopy immunostaining patterns support the localization of PGH synthase in the endoplasmic reticulum. This is the first demonstration of the ultrastructural localization of PGH synthase in the mouse embryo. Its presence before the apposition with the endometrial epithelium supports the hypothesis that arachidonic acid metabolism via the PGH synthase pathway may be crucial for implantation.
\end{abstract}

\section{Introduction}

Synthesis of prostanoids in the embryo and endometrium is involved in implantation. Prostaglandin $\mathrm{H}(\mathrm{PGH})$ synthase, also known as cyclooxygenase, is the key enzyme in the conversion of arachidonic acid to prostanoids. Arachidonic acid metabolism via the PGH synthase pathway has been demonstrated in animal embryos (Lewis et al,, 1982; Niimura and Ishida, 1987; Neulen $e$ al., 1991) and there are indications that there is a similar pathway in human embryos (Holmes et al., 1990). In addition, mRNA encoding PGH synthase was recently demonstrated in mouse embryos before apposition with glandular endometrial epithelium (Satoh et al., 1994; Takami et al., 1994).

PGH synthase is a membrane-bound enzyme, located in both the endoplasmic reticulum and the nuclear membrane (Smith and Bell, 1978; Huslig et al., 1979; Rollins and Smith, 1980). Immunohistochemistry studies, based on light microscopy, support its presence in the endoplasmic reticulum of mouse preimplantation embryos (Marshburn et al., 1990). To the best of our knowledge, these data have not been confirmed by other workers, nor have attempts at ultrastructural localization of PGH synthase in embryos been reported. A

Revised manuscript received 13 March 1996. series of immunohistochemical and ultrastructural experiments was conducted at various stages of embryonic development to establish the presence and localization of PGH synthase in preimplantation embryos.

\section{Materials and Methods}

Animals and collection of embryos and uteri

Four groups of untreated (no induction of ovulation) female mice of the Albino Swiss CF-I strain were caged overnight with male mice. The presence of a vaginal plug was used as an indication of pregnancy (day 0 of pregnancy). Pregnant mice were killed by cervical dislocation.

Embryos were collected from the first group of mice (group I) $48 \mathrm{~h}$ after observation of the vaginal plug, by flushing the oviducts and uterus with PBS through a thin injection needle introduced carefully into the tubal ampulla. Embryos were collected from a second group (group II) after $72 \mathrm{~h}$, by flushing the uterus transcervically. Embryos obtained from these two groups were at the two-cell, the four-eight-cell, the morula and blastocyst stages. In groups III and IV, the uterus and oviducts were removed for sectioning on days 2 and 3, respectively. 
Table 1. Number of flushed mouse embryos and the number of sections of mouse uteri and oviducts containing embryos examined for prostaglandin $\mathrm{H}(\mathrm{PGH})$ synthase activity at different stages of embryonic development

\begin{tabular}{lrrrr}
\hline & \multicolumn{3}{c}{ Stage of development } \\
\cline { 2 - 5 } Treatment & 2-cell & 4-8-cell & Morula & Blastocyst \\
\hline Flushed embryos treated with anti-PGH synthase & 23 & 41 & 39 & 28 \\
Control 1 (no anti-PGH synthase) & 4 & 10 & 7 & 4 \\
Control 2 (anti-actin used as control primary antibody) & 2 & 3 & 2 & 2 \\
Sections of uteri and oviducts containing embryos & 17 & 21 & 21 & 7 \\
Control 1 (no anti-PGH synthase) & 8 & 10 & 10 & 3 \\
Flushed embryos treated with anti-PGH synthase-1 & 5 & 7 & 4 & \\
\hline
\end{tabular}

Table 1 gives the number of sections and embryos examined for each stage of embryonic development.

\section{Immunohistochemical techniques for light and confocal scanning microscopy}

All tissues were immersed in $4 \%(\mathrm{w} / \mathrm{v})$ paraformaldehyde in

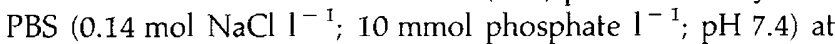
$4^{\circ} \mathrm{C}$ for $1.5 \mathrm{~h}$ (groups I and II) or $24 \mathrm{~h}$ (groups III and IV). Sections of uterus were dehydrated in graded ethanol (50$100 \%$ ) and xylene and embedded in paraffin wax for localization of embryos by light microscopy. Sections of uteri with embryos were deparaffinized in xylene and rehydrated in ethanol $(100-50 \%)$ before localization of PGH synthase. Flushed embryos were treated with anti-PGH synthase after fixation.

PGH synthase was localized using a commercially available mouse IgG monoclonal antibody (anti-PGH synthase; Cayman, Ann Arbor, MI). This antibody binds to both constitutive PGH synthase 1 and inducible PGH synthase 2 . After application of the anti-PGH synthase (10 $\mu \mathrm{g} \mathrm{IgG} \mathrm{ml}^{-1}$ in $0.05 \mathrm{~mol} \mathrm{PBS} \mathrm{l}^{-1}$ ) specimens were incubated for $20 \mathrm{~h}$ at room temperature in a humidified chamber. The sections were then washed in PBS and incubated with a second antibody, rabbit anti-mouse immunoglobulin (RAM; Dako, Glostrup), conjugated with either peroxidase or fluorescein isothiocyanate (FITC), for $1 \mathrm{~h}$ at room temperature.

Peroxidase staining. The sections of uterus and oviducts with embryos and the flushed embryos were incubated with the RAM-peroxidase complex for I h at room temperature. All specimens were washed in PBS and developed for 6 min using $0.05 \%(\mathrm{w} / \mathrm{v})$ diamino-benzidine tetrahydrochloride in $0.1 \mathrm{~mol}$ Tris maleate $\mathrm{l}^{-1}, \mathrm{pH} 7.6$, with $0.01 \%$ hydrogen peroxide. The sections were briefly counterstained with haematoxylin. Sections were rehydrated in ethanol (50-100\%) and xylene and mounted in Entellan (Merck, Darmstadt) for light microscopy. The flushed embryos were embedded in EPON 812 (Merck), after which 1-2 $\mu \mathrm{m}$ sections were made. Controls for nonspecific staining were conducted by incubation with the RAMperoxidase complex only, omitting the anti-PGH synthase step.

Fluorescein staining. Sections of uteri and oviducts with embryos and flushed embryos were incubated with RAM-
FITC for $1 \mathrm{~h}$ at room temperature. These specimens were treated with $2 \%(w / v)$ DABCO (Sigma, St Louis, USA) in $40 \%$ $(\mathrm{v} / \mathrm{v})$ glycerol in PBS, containing pyridium iodide as a nuclear stain. Nuclear staining facilitates the contrast localization of PGH synthase in the embryos and endometrium on confocal scanning microscopy.

Controls for nonspecific staining were obtained by incubation with RAM-FITC only. Nonspecific interaction between mouse antibodies and mouse tissue antigens (Hierck et al., 1994) was excluded by using anti-actin (HHF-35; Dako) as a control primary antibody instead of anti-PGH synthase.

All samples were examined under the fluorescence microscope and by confocal scanning microscopy (Bio-Rad Lasersharp MRC-600 CLSM). Stereoscopic photographs were taken during several confocal scanning sessions.

\section{Immunohistochemical detection of PGH synthase 1}

Flushed embryos were treated with anti-PGH synthase I after fixation. PGH synthase I was localized with a commercially available mouse IgG monoclonal antibody (anti-PGH synthase 1; Cayman) and RAM-FITC, using exactly the same techniques as described above for PGH synthase. All samples were examined by confocal staining microscopy and stereoscopic photographs were taken.

\section{Reflection contrast microscopy (RCM) and transmission electron microscopy (TEM)}

Flushed embryos were fixed in $4 \%$ paraformaldehyde in PBS for $1 \mathrm{~h}$. After rinsing with PBS and PBS plus $0.1 \%(\mathrm{w} / \mathrm{v}) \mathrm{BSA}-\mathrm{C}$ (BSA-C: acetylated BSA; Aurion, Wageningen) for $20 \mathrm{~min}$, the embryos were incubated overnight with anti-PGH synthase (1:200; $10 \mu \mathrm{g} \mathrm{IgG} \mathrm{ml} \mathrm{g}^{-1}$ in PBS-0.1\% BSA-C) followed by washing in PBS and $0.1 \%$ BSA-C $(3 \times 20 \mathrm{~min})$. After incubation for $7 \mathrm{~h}$ with the second antibody, goat anti-mouse (GAM, 1:30; Aurion), and conjugation with ultrasmall gold particles in PBS-0.1\% BSA-C, the embryos were rinsed in PBS $(20 \mathrm{~min}$ ) and incubated overnight in PBS. After rinsing in demineralized water $(2 \times 15 \mathrm{~min})$ a silver enhancement kit (Aurion) was used for detection ( $1 \mathrm{~h}$ incubation). After a further wash with demineralized water $(30 \mathrm{~min})$ and $0.1 \mathrm{~mol}$ cacodylate buffer $1^{-1}$ $(30 \mathrm{~min})$, the embryos were fixed for $1 \mathrm{~h}$ in half-strength Karnovsky's fixative (containing $2 \%(\mathrm{w} / \mathrm{v})$ paraformaldehyde 


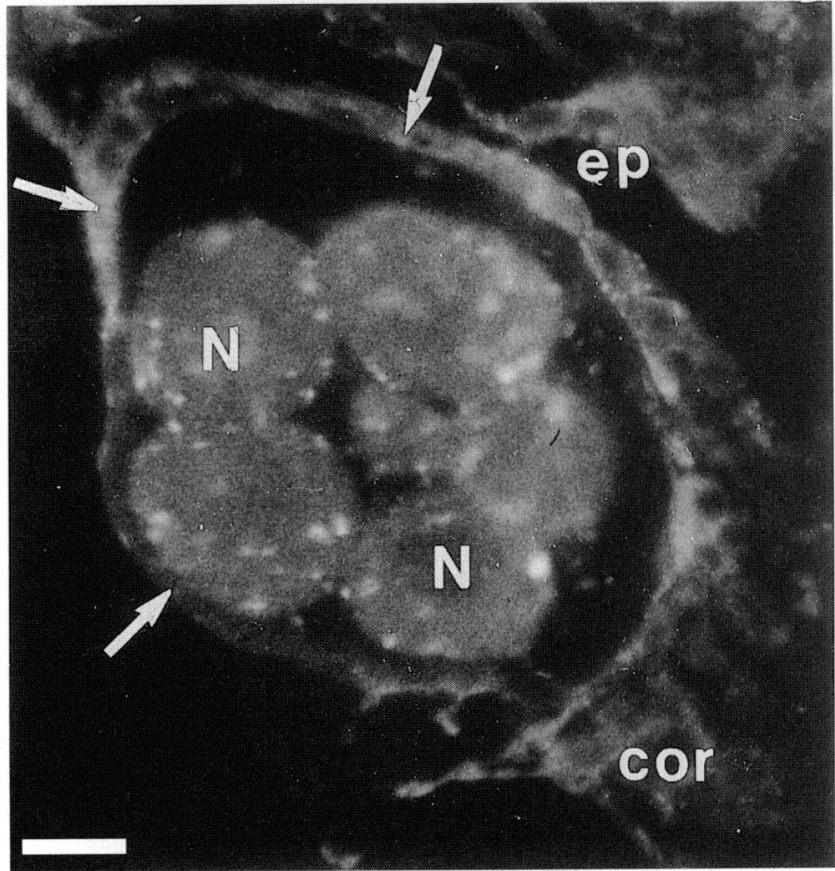

Fig. 1. Section of oviduct and embryo (eight-cell stage) incubated with anti-prostaglandin $\mathrm{H}$ (PGH) synthase and rabbit anti-mousefluorescein isothiocyanate. Fluorescence microscopy was used to observe immunostaining in the cytoplasm of the embryo and in the remnants of corona cells. Scale bar represents $10 \mu \mathrm{m}$. N: nucleus; cor: remnants of corona ceils; ep: epithelium of the oviduct. Arrows indicate the zona pellucida.

and $1.25 \%(\mathrm{w} / \mathrm{v})$ glutaraldehyde) in $0.1 \mathrm{~mol}$ cacodylate buffer $1^{-1}$. The embryos were rinsed with cacodylate buffer $(2 \times 15 \mathrm{~min})$, and then postfixed for $15 \mathrm{~min}$ in $1 \%(\mathrm{w} / \mathrm{v}) \mathrm{OsO}_{4}$ in $0.1 \mathrm{~mol}$ cacodylate buffer $1^{-1}$, followed by washing in $0.1 \mathrm{~mol}$ cacodylate buffer $\mathrm{l}^{-1}(2 \times 15 \mathrm{~min})$. The samples were then dehydrated in graded ethanol $(50-100 \%)$, embedded in EPON 812 and ultrathin sections cut.

For TEM, the ultrathin sections were contrasted with $8 \%$ $(w / v)$ uranyl acetate and lead citrate. Controls for nonspecific staining were obtained using the same method but without the anti-PGH synthase. The sections prepared for TEM were examined in a Philips CM410 electron microscope, operating at $80 \mathrm{kV}$. RCM was performed using a Leitz Orthoplan microscope (Leitz, Wetzlar) as described by Cornelese-ten Velde et al. (1988), Cornelese-ten Velde and Prins (1990) and Prins et al. (1993).

\section{Results}

All embryos demonstrated PGH synthase reactivity. Immunostaining appeared to be more intense in two-cell stage embryos, four-eight-cell stage embryos and morulae than in blastocysts. Light microscopy, fluorescence microscopy and confocal scanning of flushed embryos (sectioned only after immunohistochemistry) showed the immunostaining at the cell membrane of the blastomeres and in the zona pellucida. Use of the same techniques on sections of uterus and oviducts containing embryos, however, indicated a cytoplasmic localization of PGH synthase (Fig. 1). Cytoplasmic immunostaining was also observed in stereoscopic photographs of flushed embryos, made during confocal scanning (Fig. 2).

PGH synthase I was present in two-cell stage embryos, four-eight-cell stage embryos, morulae and blastocysts. The distribution of immunostaining was similar to the pattern observed in embryos treated with anti-PGH synthase.

With RCM and TEM of flushed embryos, in which the ultrathin sections were made after immunohistochemistry, immunoreactivity for PGH synthase was found in the zona pellucida, the cell membrane of the blastomeres and in the cytoplasm (Figs 3 and 4). In the two-four cell and morula stage embryos, abundant intracytoplasmic immunostaining was observed. Intracytoplasmic immunostaining of blastocysts was significantly less and appeared to be more intense in the trophoblast than in the embryoblast (Fig. 4). In the cytoplasm, PGH synthase appeared to be localized in the endoplasmic reticulum (Figs 3 and 4 ).

Controls for nonspecific staining, by omitting the anti-PGH synthase step, demonstrated only minimal background immunoreactivity with all methods used. No immunoreactivity was found when anti-actin (HHF-35) was used as a control primary antibody.

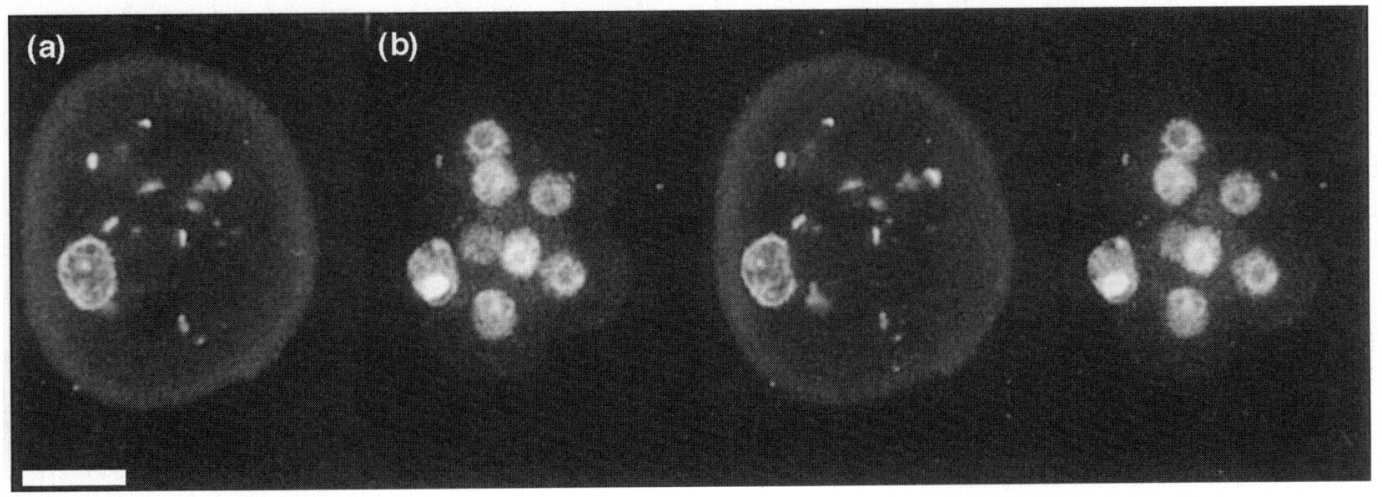

Fig. 2. (a) Stereoscopic representation of a flushed mouse embryo at the eight-cell stage incubated complete with anti-prostaglandin H synthase plus rabbit anti-mouse-fluorescein isothiocyanate. Immunostaining is intracytoplasmic. The observed condensation is caused by the polar body. (b) Nuclear staining with pyridium iodide (confocal scanning). Scale bar represents $20 \mu \mathrm{m}$. 

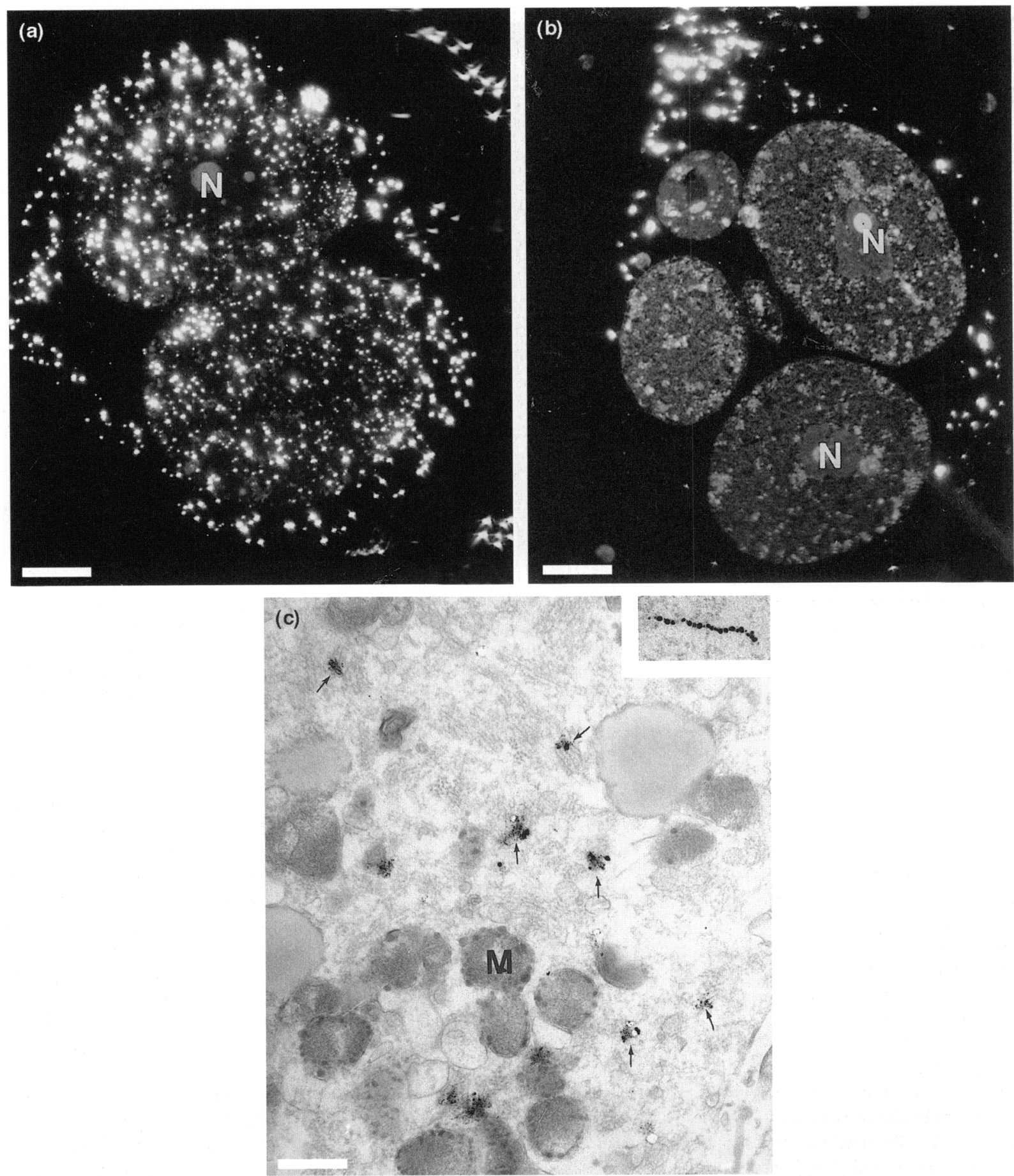

Fig. 3. (a) A flushed mouse embryo at the two-celi stage incubated with anti-prostaglandin H (PGH) synthase, goat anti-mouse antibody (GAM) and ultra-small gold particles, demonstrating anti-PGH synthase activity in the cytoplasm (reflection contrast microscopy). Scale bar represents $5 \mu \mathrm{m}$. N: nucleus. (b) A flushed mouse embryo at the four-cell stage incubated with GAM and ultra-small gold particles, demonstrating only minimal background activity and nonspecific staining in the zona pellucida (reflection contrast microscopy). Scale bar represents $5 \mu \mathrm{m}$. $\wedge$ : nucleus. (c) The same embryo as in (a) incubated with anti-PGH synthase, GAM and ultra-small gold particles with silver enhancement, demonstrating anti-PGH synthase reactivity associated with structures in the cytoplasm (transmission electron microscopy). Scale bar represents $1 \mu \mathrm{m}$. M: mitochondrion. Arrows indicate the endoplasmic reticulum). The inset shows a detail of the cytoplasm of the same four-cell stage embryo, demonstrating anti-PGH synthase activity, corresponding to localization in the endoplasmic reticulum (transmission electron microscopy). 

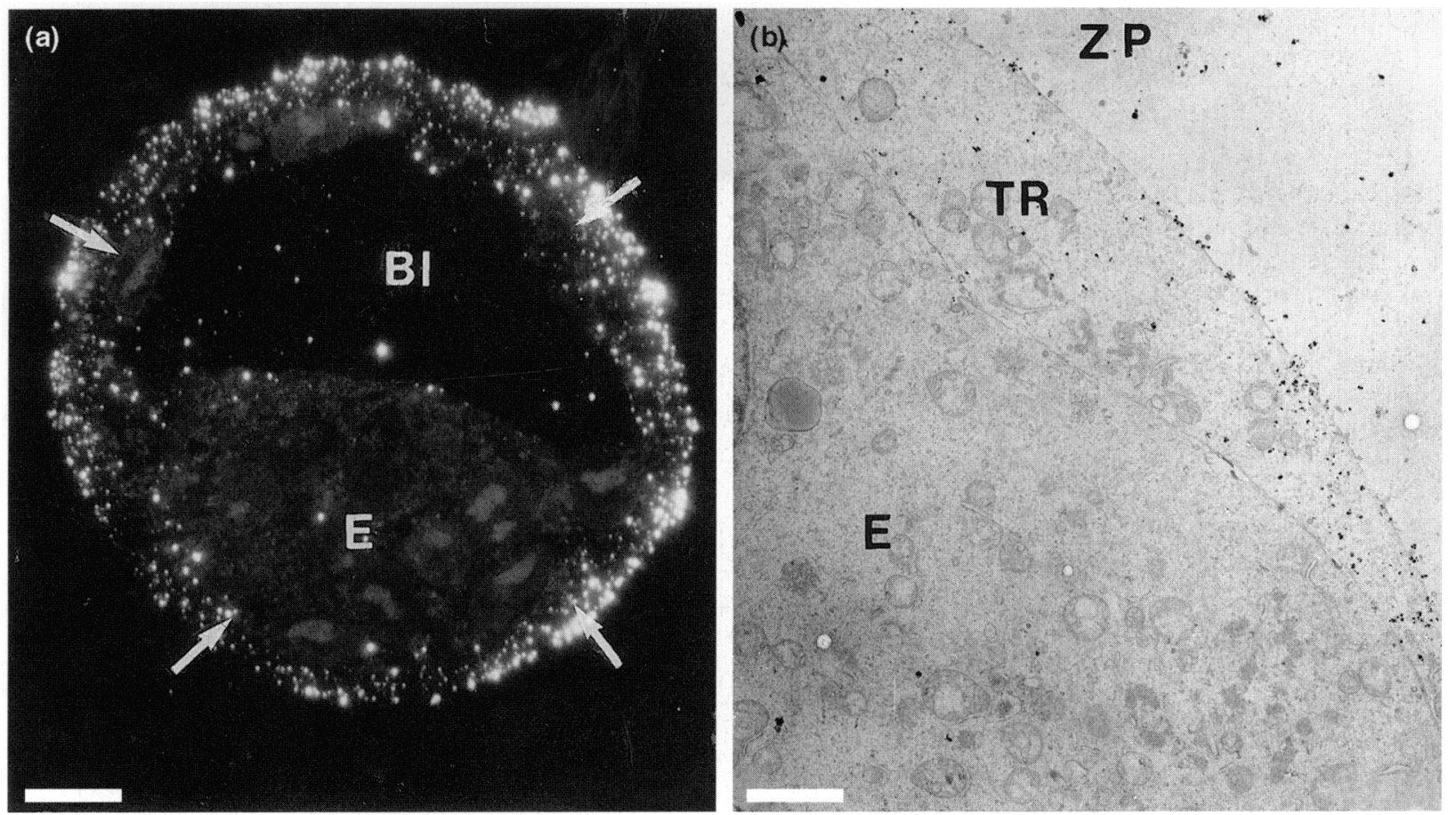

Fig. 4. (a) A flushed mouse blastocyst incubated with anti-prostaglandin $\mathrm{H}$ (PGH) synthase, goat anti-mouse antibody and ultra-small gold particles, demonstrating anti-PGH synthase activity in the cytoplasm of the trophoblast (reflection contrast microscopy). Scale bar represents $5 \mu \mathrm{m}$. E: embryoblast; BI: blastocoel. Arrows indicate the trophoblast. (b) Detail of the same blastocyst, demonstrating the distribution of anti-PGH synthase immunostaining in the trophoblast and in the embryoblast. Anti-PGH synthase reactivity is most abundant in the trophoblast (TR); only minimal (background) reactivity is observed in the embryoblast $(E)$ and zona pellucida (ZP) (transmission electron microscopy). Scale bar represents $2 \mu \mathrm{m}$.

\section{Discussion}

Several studies indicate that prostanoids affect implantation of embryos. Most studies relate to studies on prostaglandin synthesis at the site of implantation and on prostaglandin synthesis by embryos (van der Weiden et al., 1991). Immunohistochemical techniques to demonstrate $\mathrm{PGE}_{2}$ in preimplantation mouse embryos suggest that highest concentrations are found at the eight-cell and morula stages (Niimura and Ishida, 1987). When blastocysts were cultured in a medium containing $100 \mu \mathrm{mol}$ indomethacin $\mathrm{l}^{-1}$ (an inhibitor of prostaglandin synthesis), prostaglandin $\mathrm{E}_{2}$ concentrations diminished markedly (Niimura and Ishida, 1987), suggesting that the embryos themselves synthesize prostaglandin $E_{2}$. This prostaglandin has been implicated in the hatching process of blastocysts (Biggers et al., 1978; Hurst and MacFarlane, 1981), but efforts to influence hatching with indomethacin showed effects only at concentrations that were apparently toxic to embryonic development (van der Weiden et al., 1993).

Parr et al. (1988), using immunohistochemical techniques and fluorescence microscopy, demonstrated PGH synthase in rat blastocysts at days 5-7. Marshburn et al. (1990) used a peroxidase immunohistochemical method to demonstrate PGH synthase in mouse embryos present in sections of uterus and oviducts before apposition with glandular endometrial epithelium. Maximal immunostaining was observed in the cytoplasm of embryos at the morula stage (day 3 after fertilization), while immunostaining was significantly less intense after implanta- tion (blastocysts at day 5). Shemesh et al. (1994) used western blot analysis to determine PGH synthase in bovine preimplantation embryos and found PGH synthase expression in cleaved oocytes at $48 \mathrm{~h}$ but not in non-cleaved oocytes. PGH synthase expression appears to be transient and an abrupt fall in enzyme activity occurred at the early morula stage (Shemesh et al., 1994). These results indicate that PGH synthase is present at the earliest stage of embryonic development. Expression of mRNA encoding PGH synthase 1 and PGH synthase 2 has been found in early mouse embryos, and PGH synthase 1 has been detected by western blot analysis in mouse embryos at the two-cell stage (Satoh et al., 1994).

The study reported here included a range of methods from conventional light and fluorescence microscopy up to confocal scanning and electron microscopy. These methods were applied both to embryos flushed from the genital tract and to embryos identified within it. With these techniques PGH synthase activity was found in all embryos examined, thus confirming the findings of Parr et al. (1988) and Marshburn et al. (1990). In agreement with the results of Marshburn et al. (1990) and Shemesh et al. (1994), blastocysts demonstrated less PGH synthase immunostaining than did embryos at early stages of development. PGH synthase 1 activity was also present in blastomeres from the two-cell stage onwards, thereby confirming and supplementing the results of Satoh et al. (1994). Immunohistochemistry for PGH synthase 2 could not be performed since the commercially available anti-PGH synthase 2 antibody does not react with mouse PGH synthase 2 . 
Strong immunostaining of the zona pellucida and cell membrane of blastomeres was observed in the flushed embryos, but not in sectioned embryos within the genital tract. This apparent discrepancy is probably due to insufficient penetration of the antibodies or the antibody-label complex through the zona pellucida and cell membranes of the blastomeres in the embryos that were intact and not sectioned at the time of immunostaining. The zona pellucida, which consists of sulphated glycoproteins, has a fenestrated, lattice-like appearance (Yanagimachi, 1988; Nikas et al., 1994) that could have trapped some of the histochemical labels (similar fluorescence patterns, that is, immunostaining of the zona pellucida and the cell membranes of the blastomeres, have been observed in other histochemical studies of complete embryos (Thomas et al., 1994)).

Previous studies on the immunohistochemical localization of PGH synthase in other tissues located the enzyme in the endoplasmic reticulum and the nuclear membrane (Smith and Bell 1978). PGH synthase has been localized in the kidney of several mammals (Smith and Wilkin, 1977; Smith and Bell, 1978), in the ovine uterus (Huslig et al., 1979) and in mouse fibroblasts (Rollins and Smith, 1980). Both PGH synthase I and PGH synthase 2 are considered to be localized in the endoplasmic reticulum (Goppelt-Struebe, 1995).

Stereoscopic examination of the confocal scanning images indicated an intracellular, but non-nuclear, localization of PGH synthase. Tissue sections of uteri and oviducts with intracavitary embryos demonstrated a predominantly intracytoplasmic localization of PGH synthase, consistent with the findings of Marshburn et al. (1990). However, the immunostaining pattern reported here was different. The cytoplasmic circular-to-ovoid blots of staining described by Marshburn et al. (1990) were not seen but the immunostaining was more finely dispersed and consistent with a localization in the endoplasmatic reticulum, as has been observed in other types of cell (Huslig et al., 1979; Rollins and Smith, 1980). The ultrastructural methods applied to ultrathin sections of embryo at the two-eight cell stage and morulae and blastocysts, support the localization of PGH synthase in the endoplasmic reticulum.

We conclude that PGH synthase is present in the cytoplasm of the preimplantation mouse embryo and is probably localized in the endoplasmic reticulum. These findings support the contention that embryos are equipped for prostanoid synthesis, a process that may play an important role in early embryonic development and implantation.

\section{References}

Biggers JD, Leonov BV, Baskar JF and Fried J (1978) Inhibition of hatching of mouse blastocysts in vitro by prostaglandin antagonists Biology of Reproduction 19 519-533

Cornelese-ten Velde I, Bonnet J, Tanke HJ and Ploem JS (1988) Reflection contrast microscopy. Visualization of (peroxidase-generated) diaminobenzidine polymer products and its underlying phenomena Histochemistry $\mathbf{8 9}$ 141-150

Cornelese-ten Velde I and Prins FA (1990) New sensitive light microscopical detection of colloidal gold on ultrathin sections by $\mathrm{rcm}$. Combination of reflection contrast and electron microscopy in post-embedding immunogold histochemistry Histochemistry 94 61-71

Goppelt-Struebe M (1995) Regulation of prostaglandin endoperoxide synthase (cyclooxygenase) isozyme expression Prostaglandins Leukotrienes and Essential Fatly Acids 52 213-222
Hierck BP, Iperen LV, Gittenberger-de Groot AC and Poelmann RE (1994) Modified indirect immunodetection allows study of murine tissue with mouse monoclonal antibodies Journal of Histochemistry and Cyfochemistry $\mathbf{4 2}$ $1499-1502$

Holmes PV, Sjögren A and Hamberger L (1990) Prostaglandin-E2 released by pre-implantation human conceptuses Journal of Reproductive Immunology 17 $79-86$

Hurst PR and MacFarlane DW (1981) Further effects of nonsteroidal antiinflammatory compounds on blastocyst hatching in vitro and implantation rates in the mouse Biology of Reproduction 25 777-784

Huslig RL, Fogwell RL and Smith WL (1979) The prostaglandin-forming cyclooxygenase of ovine uterus: relationship to luteal function Biology of Reproduction 21 589-600

Lewis GS, Thatcher WW, Bazer FW and Curl JS (1982) Metabolism of arachidonic acid in vitro by bovine blastocysts and endometrium Biology of Reproduction 27 431-439

Marshburn PB, Shabanowitz RB and Clark MR (1990) Immunohistochemical localization of prostaglandin $\mathrm{H}$ synthase in the embryo and uterus of the mouse from ovulation through implantation Molecular Reproduction and Development 25 309-316

Neulen J, Williams RF, Schaefer W, Flecken U and Hodgen GD (1991) Arachidonic acid metabolism and prostaglandin production by primate preimplantation blastocysts Prostaglandins 42 121-125

Niimura S and Ishida K (1987) Immunohistochemical demonstration of prostaglandin E-2 in preimplantation mouse embryos Journal of Reproduction and Fertility 80 505-508

Nikas G, Paraschos T, Psychoyos A and Handyside A (1994) The zona reaction in human oocytes as seen with scanning electron microscopy Human Reproduction 11 2135-2138

Parr MB, Parr EL, Munaretto K, Clark MR and Dey SK (1988) Immunohistochemical localization of prostaglandin synthase in the rat uterus and embryo during the peri-implantation period Biology of Reproduction 38 333-343

Prins FA, van Diermen-Steenvoorde R, Bonnet J and Cornelese-ten Velde I (1993) Reflection contrast microscopy of ultrathin sections in immunocytochemical localization studies: a versatile technique bridging electron microscopy with light microscopy Histochemistry 99 417-425

Rollins TE and Smith WL (1980) Subcellular localization of prostaglandinforming cyclooxygenase in Swiss mouse 3T3 fibroblast by electron microscopic immunocytochemistry Journal of Biological Chemistry 255 4872-4875

Satoh K, Tsubata K, Sakamoto H, Tochigi M, Hayakawa S, Miyagawa Y, Takami M, Hashimoto Y, Ige S, Suzuki M, Esumi M, Tsutsumi O and Yano T (1994) Dynamic alteration of metabolism in oocyte at the perifertilization period in reference to prostaglandin involvement. In Frontiers in Endocrinology Perspectives on Assisted Reproduction pp 105-112 Eds T Mori, T Aono, T Tominaga and M Hiroi. Ares-Serono Symposia Publications, Rome

Shemesh M, Gurevich M and Harel-Markowitzl E (1994) Induction of cyclooxygenase and prostaglandin $E_{2}$ production by bovine pre-embryo Journal of Reproduction and Fertility Abstract Series 13, Abstract 126

Smith WL and Bell TG (1978) Immunohistochemical localization of the prostaglandin-forming cyclooxygenase in renal cortex American Journal of Physiology 235 451-457

Smith WL and Wilkin GP (1977) Immunohistochemistry of prostaglandin endoperoxide-forming cyclooxygenase: the detection of the cyclooxygenase in rat, rabbit and guinea pig kidneys by immunofluorescence Prostaglandins 13 873-892

Takami T, Sakamoto H, Takami M, Hayakawa S and Satoh K (1994) Cyclooxygenase in ooplasm is essential for early embryonal differentiation but not for successful fertilization in the mouse Acta Obstetrica et Gynaecologica Japonica $46295-300$

Thomas M, Jain S, Kumar GP and Laloraya M (1994) Transient thiol-expression and thiol disulphide cluster-receptor communications: a molecular basis for blastocyst-uterine interactions during implantation Biochemical and Biophysical Research Communications 198 150-157

van der Weiden RMF, Helmerhorst FM and Keirse MJNC (1991) Influence of prostaglandins and platelet-activating factor on implantation Human Reproduction 6 436-442

van der Weiden RMF, Verdijk RM, Poelmann RE, Helmerhorst FM and Keirse MJNC (1993) The influence of indomethacin on the hatching of mouse blastocysts Prostaglandins, Leukotrienes and Essential Fatty Acids 49 683-686

Yanagimachi R (1988) Mammalian Fertilization. In The Physiology of Reproduction pp 155-156 Eds E Knobil and JD Neill. Raven Press, New York 